simplest possible construction and the straight catheters are those generally used. The lithoclast is also Mr. Milton's pattern and is to be used through a comparatively large wound.

Cairo.

\section{SOME OBSERVATIONS POINTING TO AN INTRACORPUSCULAR STAGE OF DEVELOPMENT IN THE TRYPANOSOME.}

BY E. J. MOORE, F.R.C.S. IREL.,

WISTRICT MEDICAL OFFICER, ASABA, SOUTHERN NIGERIA, WEST AFRICA.

ON making daily examinations of the blood from the peripheral circulation of cattle suffering from a severe trypanosoma infection I noticed free in the plasma numerous exceedingly minute spherical coccus-like bodies of a highly refractive nature. These bodies soon after their first appearance united in pairs and then became exceedingly active, the movements consisting principally of rapid flexion and extension of the combined pair and a slight absolute movement through the field of observation. 24 hours after their first appearance these bodies were seen to be no longer free but attached in large numbers along the periphery of the red corpuscles, slightly indenting their substance. On the third day the bodies, now almost completely intracorpuscular, had become very much larger and elongated to a peg-shape, the head lying in the body of the corpuscle while the shank projected from its surface, being evidently inclosed in a prolongation of the stroma which formed a sheath from which the point of the intracorpuscular body was sometimes protruded like the sting of an insect and sometimes suddenly retracted, the whole body travelling inwards through the substance of the corpuscle and impinging on its opposite wall. The projecting portions of these peg-shaped bodies, sometimes curved and sometimes straight, were in constant motion, the tips were blunt and evidently from their behaviour were provided with flagella, which, however, I was unable to perceive. In addition to these elongated bodies others of a spherical form were present moving actively inside the corpuscles and occasionally pushing the corpuscular substance outwards, elongating and appearing as projections from the surface; the condition was so general that each corpuscle contained from two to six of the bodies which gradually increased in size and gave to each individual disc an independent motion of a very interesting nature. The corpuscle could sometimes be seen to communicate a motion to its neighbour, with which it was not actually in contact, a phenomenon due, in my opinion, to the presence of flagella in the tips of the projecting peg-shaped bodies; at other times these bodies gave one the idea of trying to pull themselves free of the corpuscles and by their efforts individual corpuscles were pulled out of shape, sometimes appearing as ovals with a prolongation from each extremity from which protruded the tip of a peg-shaped body; at other times, when three of these bodies were present, the shape assumed was roughly triangular and constantly varied according to the strength of pull exerted by the contained peg-shaped bodies.

On the eighth day after the appearance of the free coccoid bodies in the plasma the intracorpuscular bodies in some cases separated to one side of the corpuscle, the hæmoglobin being collected to the other side; they then became snailshaped and a marked constriction appeared between them and the bodies of the corpuscles, presenting the appearance of being budded off. After remaining for about 24 hours in this condition I have observed them to become detached and float free with a sluggish movement in the plasma, being then in shape, appearance and size remarkably like small trypanosomes but without sheath or spot. On the tenth day the blood of the animals, hitherto free from trypanosomes, was found to contain them in immense numbers and two days later the coccoid bodies which had been absent since the stage in which $\mathrm{I}$ have described them as invading the corpuscles again began to swarm in the plasma, and the cycle, which thus was completed in 10 days, ecommenced.

On the appearance of the trypanosomes I made prolonged examination of the blood with the view to discover their mode of reproduction and was unable to find in any case evidence of longitudinal fission. I noticed, however, that the parasites were very often found in pairs and observed them travelling across the slide towards one another; the parasites also exhibited motions of a very peculiar nature, the body substance becoming repeatedly retracted to either extremity and sometimes at the same time to both extremities, forming one or two spheres with a bright spot in the centre. These movements were sometimes followed by the death of the parasite and it is a matter of interest that in dying the animal invariably assumed a globular shape; this probably accounts for the difficulty in finding trypanosomes post mortem even when they have caused the death of an animal. (That trypanosomes do not alter their shape in dried smears is due, $\tilde{I}$ believe, to the fact that the process of drying fixes the animal in position before it has time to assume the circular shape.) In the cases of parasites which survived longer there was often a definite series of movements of the posterior portions of the body substance to the extremity of the tail, the tail-spot being jerked along with the protoplasm, which then gradually returned to its original position, the tail-spot remaining stationary against the side of the sheath while the protoplasm rippled forward past it ; the result of the movements was that the tail-spot became slowly pushed towards the hinder extremity and though I cculd not convince myself that I ever saw the spot being actually extruded all the movements of the parasite seemed to be purposive and directed towards getting rid of the tail-spot which seemed to constitute a source of irritation.

There is one further point-namely, that the time of the appearance of the coccoid bodies in the plasma coincided with those movements of the tail-spot and that, when under the conditions described above, a free coccoid body and a trypanosoma appeared in the same field the tail-spot and coccoid body showed such a remarkable similarity that their optical characteristics were in fact identical.

From the description by Major C. Donovan, I.M.S., which I have read in THE LANCET of Sept. 10th, p. 744, of the piroplasmata Donovani I am inclined to think that the intracorpuscular peg-shaped bodies are of a similar nature to those which he describes. From my observations it will thus be seen that minute spherical bodies will sometimes be found periodically swarming in the plasma in animals infected with trypanosomes; that these bodies unite in pairs, appearing as comma-shaped organisms with highly refractive extremities; that these bodies later invade and penetrate the red corpuscles, becoming peg-shaped and increasing greatly in size; and that they finally take on a snail-shaped appearance and are extruded into the plasma. The extrusion of the snail-shaped bodies being followed by the appearance of large numbers of trypanosomes and the peculiar movements of the tail-spot being coincident with the presence of the free coccoid bodies would also seem to indicate that in the blood under observation the reproduction of the trypanosomes was effected by the extrusion of the tail-spot which, after undergoing development inside the red corpuscles, eventually became again free in the plasma and there possibly acquired the remaining characteristics of the mature organism.

Southern Nigeria.

\section{Clinital athotes:}

\section{MEDICAL, SURGICAL, OBSTETRICAL, AND THERAPEUTICAL.}

\section{NOTE ON A CASE OF INTUSSUSCEPTION.}

By J. McDonaLd, M.D. EdIN.

ON Feb. 17th, 1903, I was called to a house about one and a half miles distant. On my arrival at noon I found a male child, aged 16 months, suffering from abdominal pain and vomiting and passing blood-stained mucus per anum. These symptoms had come on suddenly an hour previously. On abdominal examination a tumour was detected in the upper portion of the right inguinal region. This tumour was 